\title{
Cambios ambientales holocénicos en la Puna de Atacama y sus implicancias paleoclimáticas
}

\author{
Lautaro Núñez ${ }^{1}$, Martin Grosjean ${ }^{2},{\text { Bruno Messerli }{ }^{2} \text { y Hans Schrelier }}^{3}$
}

\begin{abstract}
RESUMEN
Estudios multidisciplinarios han permitido reconstruir los cambios extremos en el balance hídrico experimentados en la Puna de Atacama durante el Holoceno. La intensificación del monzón de verano (invierno boliviano) aumentó la precipitación de origen continental en la región hasta los $25^{\circ} \mathrm{S}$ durante el Tardiglacial/Holoceno Temprano. La precipitación en la región de los Andes occidentales $\left(24^{\circ} S\right)$ se incrementó hasta $500 \mathrm{~mm}$ por año en comparación con los $200 \mathrm{~mm}$ anuales de la actualidad. Durante el período entre 8400 y 3000 años AP, aproximadamente, la extrema aridez y la escasa precipitación dominada por tormentas muy intensas pero esporádicas fueron responsables de un descenso dramático del nivel de los lagos. A partir de los 3000 años AP, el cinturón de lluvia tropical volvió a desplazarse hacia el sur en varias fases hasta su posición actual (isoyeta de $200 \mathrm{~mm}$ anuales a $24^{\circ} \mathrm{S}$ ). Diversos factores se consideran para explicar esta evolución paleoclimática: teleconexiones con el hemisferio norte, cambios en la circulación oceánica en el Pacífico oriental, cambios ambientales en la cuenca amazónica donde se origina el vapor de agua, o diferencias en albedo (radio de entrada y salida de la radiación solar) y cubierta de nubes en la región de Atacama. Estos estudios son indispensables para atender la problemática de la adaptación humana a los ambientes finipleistocénicos y holocénicos del Desierto de Atacama.
\end{abstract}

\begin{abstract}
Strengthened sununer monsoon brought tropical/continental moisture as far south as $25^{\circ} \mathrm{S}$ during late-glacial and early Holocene times. Precipitation rates in the Altiplano of the western Andes $\left(24^{\circ} \mathrm{S}\right)$ increased to $500 \mathrm{~mm}$ yr-1 compared to $<200 \mathrm{~mm}$ yr-1 today. There is evidence of dramatically decreasing lake levels belween 8400 and about 3000 yr BP, and conditions drier than today were established. This arid period was interrupted by low-frequency but heavy storms. The monsoon precipitation belt advanced once again in several stages to its current position (200 $\mathrm{mm}$ yr-1 isohyetaat $24^{\circ} \mathrm{S}$ ) around $3000 \mathrm{yr} \mathrm{BP}$. The reasons for these changes the variable circulation in the E-Pacific, teleconnection to the northern hemisphere, environmental changes in the source área of the moisture (i.e. tropical continent), or internal forcing
\end{abstract}

1 Instituto de Investigaciones Arqueológicas y Museo, Universidad Católica del Norte, Casilla 17, San Pedro de Atacama, CHILE.

2 Department of Physical Geography, University of Bern, 12 Hallestrasse, CH-3012 Berna, SUIZA.

3 Resource Management Science, University of British Columbia, Vancouver BC V6T 1Z3, CANADA. due to changes in the radiation budget of the Altiplano área considered as possible explanations. Relationship beetwen archaic occupation and late Pleistocene-Holocene time are preliminary considered.

\section{Introducción}

El régimen holocénico es conocido como el período climático más estable durante los últimos 150.000 años (Lorius y Oeschger 1994). Esta opinión se basa en los registros de $\delta^{18} \mathrm{o}$ en los sondajes de hielos localizados en las altas latitudes de los hemisferios norte y sur en referencia principalmente a los cambios de temperatura. Nuevas evidencias provenientes de las áreas tropicales reconsideran esta opinión en torno a un Holoceno "estable", porque aquí los cambios reflejan dramáticas variaciones en el régimen de humedad. En las áreas tropicales del hemisferio norte, el Holoceno Pluvial Temprano es atribuido a la intensificación del monzón de verano, principalmente impulsado por el aumento de la intensidad de la insolación de verano (Kutzbach y StreetPerrolt 1985). Se han descubierto evidencias para explicar los cambios abruptos en las precipitaciones durante los últimos 10000 años en la parte tropical de los Andes Centrales (p.e., Lago Titicaca) y en los Andes peruano-bolivianos (Thompson et al. 1985; Martin et al. 1993 y otros), incluyendo Brasil central (Ledru 1993), y en la franja de precipitaciones invernales del Chile Central entre los $30^{\circ} \mathrm{S}$ y $32^{\circ} \mathrm{S}$ (Villagrán y Varela 1990; Veit 1996). Al respecto, se ha especulado que la ENSO desempeñó un papel importante en el control del patrón de precipitaciones en los Andes tropicales y subtropicales. Sin embargo, poco se sabe sobre las causas de tales cambios en términos de humedad a gran escala, a largo plazo, y de las fluctuaciones de la corriente de Humboldt en esta área.

La llamada Puna de Atacama ocupa las tierras altas de la II Región del norte de Chile (Figura 1). Se localiza actualmente en una zona extremadamente árida, donde la franja de lluvias del verano tropical y la de las precipitaciones del invierno extratropical 


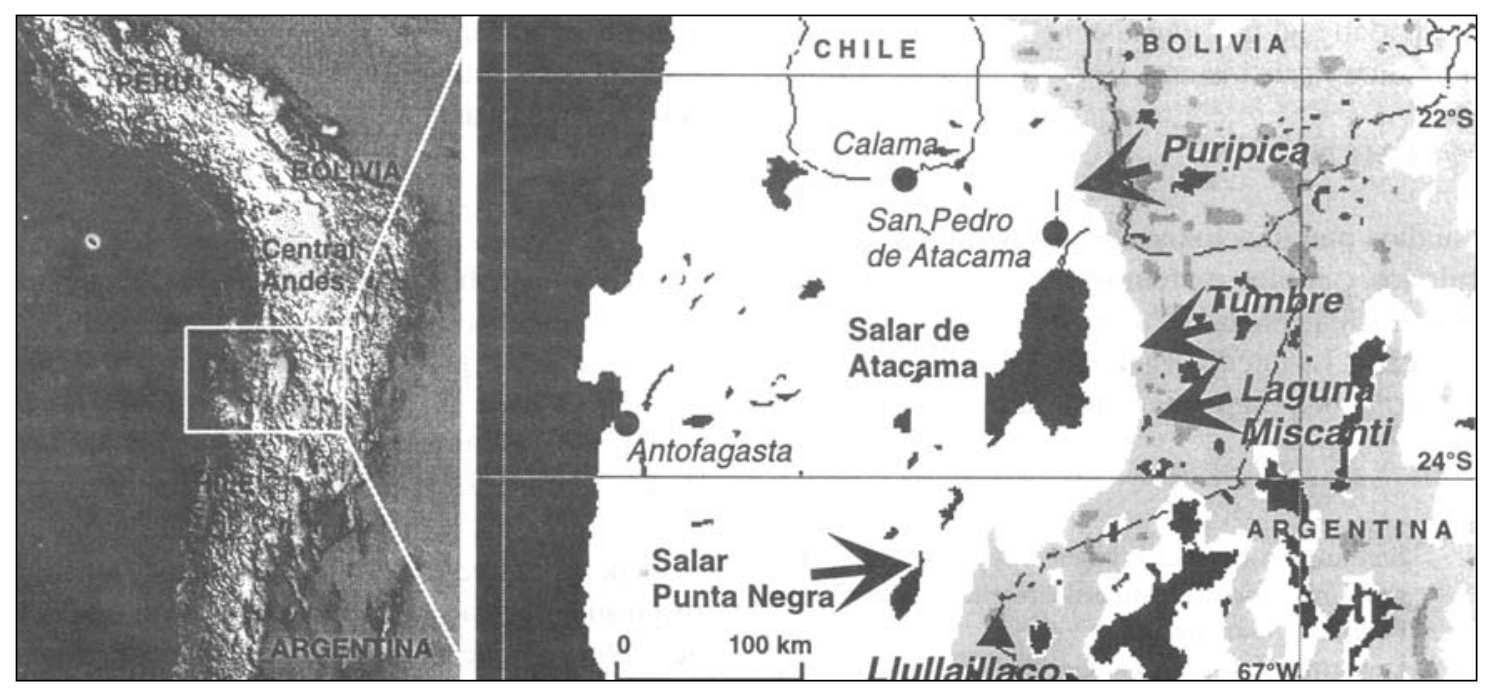

Figura 1. Mapa del área de investigación. El sector sombreado se ubica sobre los $4000 \mathrm{~m}$. La figura de la izquierda proviene de USGS-DEM e Isacks (1988).

convergen y algunas veces se sobreponen. Esta región es un desierto de altura, ubicada entre los $19^{\circ} \mathrm{S}$ y $\operatorname{los} 27^{\circ} \mathrm{S}$ de naturaleza tan árida que actualmente no podría mantenerse ningún glaciar. Sin embargo, es altamente sensible a las variaciones en términos de humedad efectiva y es por lo tanto una de las áreas claves para estudiar los ajustes y/o las intensidades cambiantes de la faja de circulación tropical y occidental y conducir así el fenómeno de los cambios de humedad en los Andes tropicales durante el régimen holocénico.

La mayoría de las evidencias paleoclimáticas de la Puna de Atacama documentan una fase tardía-glacial húmeda reconocida como Tauca. Las superficies de los lagos entre los $21-24^{\circ} \mathrm{S}$ aumentaron dramáticamente y se establecieron grandes espejos de agua dulce en las tierras altas entre $c a .15000 \mathrm{y}<10400$ años AP. Modelos de balance energético e hídrico de paleolagos sugieren que el rango de las precipitaciones aumentó hasta $500 \mathrm{~mm}$ al año, comparado con los <200 mm anuales de hoy (Grosjean 1994; Grosjean et al. 1995). Sin embargo, poco se sabe acerca del Holoceno, ya que la mayoría de los depósitos sedimentarios lacustres fueron destruidos. Otros archivos como los depósitos glaciales no se han fechado; por otro lado, el registro de turbas no siempre abarca el total de un período de tiempo, de modo que ciertos cambios ambientales fueron deducidos de la historia de la ocupación humana y del uso de recursos correlacionados (Grosjean y Núñez 1994).
Dentro de la problemática referida, primero describiremos la información proveniente de las nuevas fuentes que proporcionan conocimiento multidisciplinario acerca de las condiciones ambientales del Holoceno en la Puna de Atacama. Segundo, discutiremos brevemente las relaciones espaciales con el medio en las zonas circundantes. Tercero, revisaremos brevemente los patrones climáticos modernos para descubrir situaciones análogas que se asemejen espacialmente a datos de multiaproximación establecidos en el pasado. ¿Podemos explicar las condiciones del pasado, haciendo variar la frecuencia estadística de los patrones modernos? Finalmente, especularemos acerca de los posibles mecanismos de fuerza para comprender los cambios de humedad durante el Glacial Tardío y el Holoceno.

\section{Evidencias de cambios durante el Holoceno}

El fin de la fase húmeda Tauca

El fin de la fase húmeda Tauca fue el primer cambio ambiental importante en el Holoceno. Edades de radiocarbono convencional sobre estromatolitos, Ciorg total y fracciones de carbonatos de sedimentos lacustres laminados son por ahora la única información disponible. Se ha sugerido que los altos niveles lacustres y el clima húmedo del Glacial Tardío persistieron más allá de los 10400 años AP (Grosjean 1994). Condiciones tan áridas como aquéllas que se establecieron alrededor de 8400 años AP habrían permitido 
efectivamente un tiempo más o menos largo para un régimen transicional.

Esto se debe en parte a las dificultades que tuvimos con el material que usamos para fechados radiocarbónicos y que tienen que ver con el efecto reservorio (Grosjean et al. 1995). Nuevos fechados de radiocarbono en turbas del salar de Punta Negra cubren el período desde $10290 \pm$ años $\mathrm{AP}\left(\delta^{13} \mathrm{C}=\right.$ 25.5\%o, Hv-19692), 9885 \pm 300 años AP $\left(\delta^{13} \mathrm{C}=\right.$ $26.5 \%$, Hv-19694) a $8450 \pm 765$ años AP $\left(\delta^{13} \mathrm{C}=\right.$ 27.2\%o, Hv-19693; Messerli et al. en prensa). Nuestro perfil de sedimentos de $120 \mathrm{~cm}$ de profundidad (Figura 1, 24 ${ }^{\circ} 25^{\prime} \mathrm{S} / 68^{\circ}$ 54' O, $2980 \mathrm{~m}$ ) consiste en estratos alternados con turbas y depósitos de agua poco profundas (principalmente diatomitas y arcilla), sugiriendo lagos pequeños o lagunas con aguas abiertas, desagües de ríos altos provenientes de la zona del volcán Llullaillaco, por lo general en condiciones bastante húmedas. El hecho de que tales condiciones llegaran hasta muy avanzado el Holoceno ( $\tan$ reciente como los 8500 años AP), hace que en el período de transición desde la "modalidad Tauca" húmeda hasta la "modalidad del Holoceno Medio", totalmente árida, de clima bastante seco, hayan ocurrido cambios sensiblemente abruptos. Este descubrimiento sustentaría con fuerza la hipótesis de que en el vacío regional en términos de ocupación humana ("silencio arqueológico") entre los 8500 y 4800 años AP, a lo largo de la Puna occidental se inició a raíz de un dramático descenso de los recursos debido a las condiciones climáticas áridas (Grosjean y Núñez 1994). Precisamente, la identificación de ecorrefugios durante el Holoceno Medio se ha priorizado en las prospecciones actuales a través de las investigaciones en curso.

Aridez y flujos de tormentas durante el Holoceno Medio

Hasta ahora es escasa la información multidisciplinaria acerca del período extremadamente árido del Holoceno Medio (8400 hasta alrededor de los 3000 años AP). Los niveles lacustres eran extremadamente bajos, la mayoría de las cuencas estaban totalmente secas, y los sedimentos estaban sometidos a condiciones subaéreas, destruidos o erosionados por el viento. Un perfil con sedimentos y polen registrado en Tumbre (Graf 1992) es por ahora el único archivo disponible. Aquí identificamos una matriz arenosa con escasa materia orgánica entre los 7500 y 3900 años AP. Las turbas sólo llegaron a dominar después de los 3900 años AP. Por otro lado, una nueva información de sedimentos lacustres proveniente de la laguna Miscanti y sedimentos fluviolacustres localizados en la quebrada de Puripica, nos proporcionaron un registro más detallado sobre las condiciones ambientales y climáticas, válido para el Holoceno Medio (Figuras 1 y 2).

La acumulación de sedimentos fluviolacustres del Holoceno Medio ubicado en quebrada de Puripica lo hemos interpretado como una evidencia del proceso de aridez en aumento, cuando la vegetación era escasa, el control de la erosión era muy débil y la frecuencia de lluvias era muy variable (a veces entre escasa y abundante). Los sedimentos proporcionaron evidencias de tormentas muy intensas, pero esporádicas (período de retorno alrededor de 1200 a 500 años) por los 5080 años AP, un poco antes de los 3800 años AP y alrededor de los 3300 años AP. Se distinguen tres períodos de sedimentación. Entre los 6200 y los 5100 años AP no existen indicadores de grandes eventos torrenciales. Evidencias de arena fluvial y depósitos lacustres fueron dominantes. La acumulación de sedimentos y la falta de superficies erosivas sugieren bajos niveles de energía liberada en el río. Esto sería característico para un clima generalmente árido con tormentas esporádicas leves, cuando los flujos de escombros se inyectaron en el curso del río y dieron origen a un régimen de sedimentación lacustre a corto plazo. La actividad de tormentas llegó a su máximo entre los 5100 y 3800 años AP, los sedimentos de los eventos torrenciales fueron dominantes, mientras que la arena fluvial y los depósitos lacustres escasearon. Aquí hemos identificado sedimentos de por lo menos 12 pequeñas tormentas (tamaño máximo de $10 \mathrm{~cm}$ de partículas), más de 20 moderadas $(10-20 \mathrm{~cm}$ de partículas) y 2-4 grandes tormentas (partículas $>20 \mathrm{~cm}$ ). Las condiciones ambientales generales probablemente alcanzaron su punto máximo de aridez durante este período. Entre los 3800 y 3100 años AP los depósitos lacustres volvieron a ser dominantes, aunque se vieron interrumpidos por eventos torrenciales episódicos (tormentas pequeñas, moderadas y una grande). Por lo general, las condiciones secas con acumulación de sedimentos prevalecieron hasta 3100 años AP. Aunque el número y la frecuencia de los eventos mencionados aquí son, a propósito, conservadores y probablemente de baja estimación, entregan una idea concreta acerca de las dramáticas inundaciones y cambios morfodinámicos en un medio extremadamente árido.

Evidencias adicionales de condiciones más secas que las actuales fueron descubiertas en superficies 
erosivas del Holoceno Medio y las facies con exposición subaérea en los sedimentos de laguna Miscanti (ver Figura 2), sugieren un nivel de hasta $10 \mathrm{~m}$ inferior al de hoy (Valero-Garcés et al. 1996), en consecuencia con los datos de laguna del Negro Francisco localizada a los $27^{\circ} \mathrm{S}$ en las tierras altas de Copiapó (Grosjean et al. 1997).

\section{El establecimiento del clima moderno}

La transición de las condiciones extremadamente secas del Holoceno Medio a un clima moderno, un tanto más húmedo con menos de $200 \mathrm{~mm}$ de precipitaciones al año se aprecia mejor en los sedimentos de la laguna Miscanti (Valero-Garcés et al. 1995; ver Figura 2).

Los sedimentos del lago muestran a una profundidad de 180 a $145 \mathrm{~cm}$ el cambio de un ambiente superficial o poco profundo, altamente salino y con precipitación de aragonita a condiciones modernas con depósitos en un lago de $9 \mathrm{~m}$ de profundidad de agua relativamente dulce (6.4 $\mathrm{mS} \mathrm{cm}-1$ ), precipitación de calcita magnésica y abundante ópalo de diatomea. Enfatizamos la naturaleza cambiante de esta transición, sugiriendo que los cambios en el lago fueron forzados por varios estímulos de humedad, y no se debieron a una razón única. La medida del tiempo de esta transición en los sedimentos de la laguna Miscanti es aún incierta. Dado el grado actual del conocimiento paleoambiental del área interpretamos esta transición como sincrónica a aquella entre el Holoceno Medio y el Tardío de la quebrada de Puripica. Allí, el medio ambiente en el río cambió del modo de acumulación del Holoceno Medio (clima totalmente árido) al modo del patrón de erosión del Holoceno Tardío (un poco más húmedo), poco después de los $3110 \pm 70$ años AP. Esto a su vez se interpreta como un cambio significativo en el régimen hidrológico del río. $\mathrm{Al}$ respecto, sugerimos un desagüe mayor, mejor control de la erosión en las laderas adyacentes debido a una vegetación más densa, una energía liberada superior en el río, y consecuentemente una erosión lineal en el lecho. Se postula un clima por lo general más húmedo con precipitaciones modernas ( $<200 \mathrm{~mm}$ al año) y un régimen de precipitaciones un tanto más suave. Sin embargo, el potencial erosivo del río Puripica fue sorprendente en términos de que más de $16 \mathrm{~m}$ de sedimentos fueron surcados dentro de los últimos 2000 años. Obviamente, una sorprendente cantidad de agua fue requerida para estos fines y podríamos especular si las precipitaciones y el desagüe actual serían suficientes para lograr efectos similares.

Por otra parte, el análisis de los sedimentos de la laguna del Negro Francisco presenta una historia ambiental similar esta vez en la puna sureña de Copiapó. Por lo tanto, los resultados para la laguna Miscanti posiblemente dan cuenta de un patrón más regional significativo in toto (Grosjean et al. 1997).

El hecho de que la recuperación lacustre en Miscanti ocurra bajo condiciones modernas por los 3200 años AP, en plena sincronía con el inicio de la fase agropastoralista Tilocalar, localizada precisamente en el transecto Miscanti-Tulan-Tilocalar, pone de manifiesto una estricta correlación entre la emergencia de condiciones húmedas favorables y el inicio de prácticas económicas de horticultura y crianza de camélidos dominante, tema relevante que será tratado en otro estudio interdisciplinario específico (Núñez 1992).

\section{El patrón espacial de los cambios climáticos del Holoceno}

La historia climática del Holoceno observada desde tres zonas distintas en diferentes latitudes a lo largo de los Andes se muestra en la Figura 3. La zona del lago Titicaca en los $16^{\circ} \mathrm{S}$ en el régimen de precipitación tropical (Martin et al. 1993), la Puna de Atacama a los $24^{\circ} \mathrm{S}$ en la zona árida y la región costera de Chile Central por los $32^{\circ} \mathrm{S}$ y de las precipitaciones invernales de los weste-lies (cinturón de vientos del oeste o "invierno boliviano") (Villagrán y Varela 1900).

La Puna de Atacama muestra por los $24^{\circ} \mathrm{S}$ una historia ambiental similar a la del área tropical del lago Titicaca. Los resultados preliminares muestran que las condiciones totalmente áridas se establecieron un poco antes, en los $24^{\circ} \mathrm{S}$ de la Puna de Atacama que en los $16^{\circ} \mathrm{S}$, mientras que el cambio del Holoceno Medio más árido al clima moderno se produjo probablemente un poco más tarde, por los $24^{\circ} \mathrm{S}$, en relación con la cuenca del Titicaca. Se sugiere que el retroceso y el reavance de la humedad explicaría tal patrón de humedad. Sin embargo, los mecanismos y la medición del tiempo deben estar aún así mejor documentados. El aumento de la aridez en la zona de las precipitaciones de invierno, por $\operatorname{los} 32^{\circ}$, alrededor de los 10000 años AP, ocurrió unos 2000 años antes que en las zonas tropicales (Villagrán y Varela 1990). 


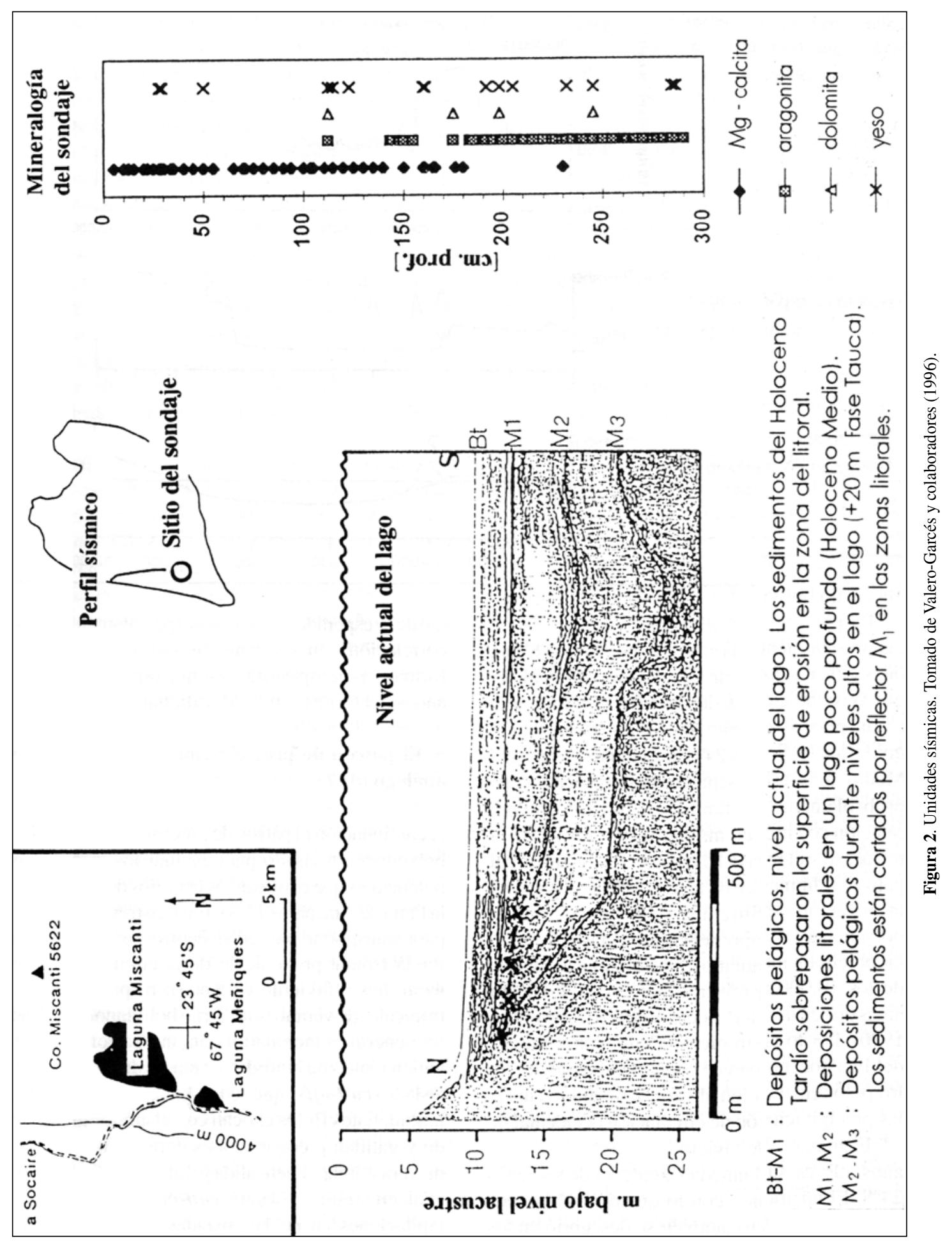




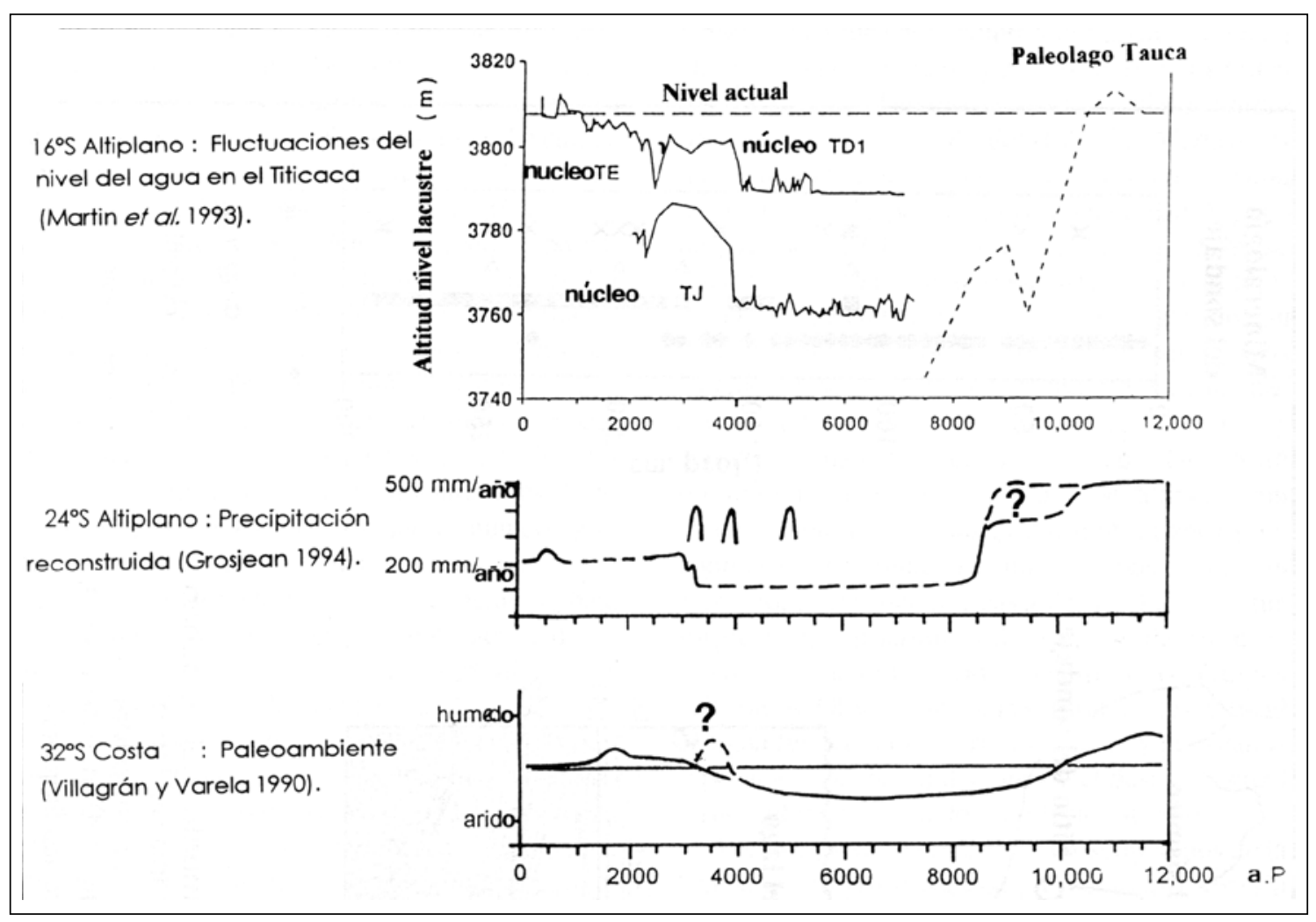

Figura 3. Gráficos.

Por lo tanto, parece ser un fenómeno del fin del Pleistoceno, relacionado con los cambios hacia los polos en el cinturón de circulación occidental de las precipitaciones de invierno.

El aumento de la humedad alrededor de los 3000 años AP es sincrónico con lo que ocurrió por los $24^{\circ} \mathrm{S}$. El mismo efecto también se descubrió en los Andes argentinos entre los $32-35^{\circ} \mathrm{S}$ (Markgraf 1983). Este es un aspecto paleoambiental más regional de lo esperado que guarda especial interés por su correlación con las primeras innovaciones protoformativas agropastoralistas que repercutirán en los Andes Centro Sur y Meridionales (Núñez 1992).

\section{El patrón de precipitaciones modernas: ¿Un análogo útil?}

A continuación el patrón de precipitación actual será brevemente revisado para evaluar los mecanismos potenciales que explican los cambios de humedad en la Puna de Atacama ( $\left.24^{\circ} \mathrm{S}\right)$. Existen tres mecanismos para transportar humedad dentro de esta zona: a) lluvia frontal proveniente de la circulación de los weste-lies ("invierno chileno"); b) precipitaciones tropicales de verano ("invierno boliviano"), aquí por lo general relacionado con un patrón de flujo anticiclónico de altitud y, c) masas de aire polar frío aislado (cutt-offs) que se trasladan en zonas de baja latitud. Estos flujos chocan con el aire cálido/ húmedo y gatillan precipitaciones durante invierno, primavera y otoño (Fuenzalida y Rutland 1986; Messerli et al. en prensa). Eventos cutt-offs que activan precipitaciones en celdas aisladas, en zonas de baja latitud y las precipitaciones de los casos analizados muestran una composición isotópica de origen continental.

El patrón de la lluvia frontal de invierno proveniente del occidente no corresponde a la información paleoambiental establecida para la Fase Tauca del Glacial Tardío/Holoceno Temprano puneño por los $24^{\circ} \mathrm{S}$. Un aumento en dirección hacia el sur del rango de lluvia podría esperarse, sin embargo, contrasta con las gradientes de humedad N-S como se deduce de las líneas de equilibrio de las antiguas glaciaciones y depósitos lacustres tardío-glaciales entre $\operatorname{los} 18^{\circ}$ y $29^{\circ} \mathrm{S}$ (Messerli et al. 1993; Messerli 
et al., en prensa). El aumento de las precipitaciones en invierno también sugeriría cambios ambientales en las zonas de menor altitud, hacia la costa del Desierto de Atacama. De acuerdo a nuestro conocimiento, se indica que los cambios de humedad ocurrieron exclusivamente en las zonas de gran altura, a lo largo de las tierras altas, mientras que las condiciones totalmente áridas prevalecieron en las zonas de baja altura.

El aumento de las precipitaciones tropicales de verano coincide con la información paleoambiental establecida para la época del Glacial Tardío y Holoceno Temprano. En efecto, el esperado aumento de humedad hacia el norte se observa en la gradiente de los niveles de antiguos lagos y las líneas de equilibrio de glaciares reconstruidos en esta zona (Messerli et al. en prensa). Sin embargo, en esta zona existió sincronía entre la glaciación máxima con la Fase Tauca (Clayton y Clapperton, en prensa). La fase húmeda Tauca tocó exclusivamente a las zonas de gran altura en los Andes, mientras que las zonas de baja altitud, inferior a los $3500 \mathrm{~m}$, fueron afectadas a lo más por grandes desagües. Esta situación es típica del régimen de precipitaciones de verano, dando una evidencia adicional en el sentido que la intensificación del monzón de verano cumplió un papel importante durante este período. Dado el conocimiento que se tiene sobre las condiciones climáticas actuales, el continente tropical es la fuente principal donde se forma el vapor de agua (Aravena et al. 1989; Grosjean et al. 1995). Es decir, el patrón moderno o actual de precipitaciones de verano, aunque intensificado, pudo también ser un modelo análogo para la circulación durante el Holoceno Temprano. El origen continental del vapor de agua implica además que se debería considerar el cambio en los regímenes de humedad en la zona en donde se origina el vapor de agua para explicar el transporte de humedad variable hacia la Puna de Atacama. Realmente, la historia ambiental de las tierras bajas del trópico (p.e., Brasil Central; Ledru 1993) se iguala notablemente a los cambios ambientales en el altiplano. Se reitera con estos datos en Sudamérica continental, nuestro énfasis en el rol de la vegetación pasada o antigua, los regímenes de desagües o deshielos y los rangos de evapotranspiración (p.e., el reciclaje del vapor de agua), para explicar los cambios de humedad durante épocas pasadas o lo largo de la Puna de Atacama.

Estas consideraciones son muy importantes a la luz de los estudios previos como el de Shukla y colaboradores (1990) quienes utilizaron un modelo de circulación global y vegetación para demostrar en el Amazonas el reemplazo hipotético de la selva por praderas como resultado del $26 \%$ de la disminución de las precipitaciones en esta zona. De esta manera, el papel crucial de la vegetación con respecto al transporte de humedad atmosférica es evidente y requiere de urgente investigación.

Los eventos cutt-offs son aspectos sinópticos interesantes y merecen más consideración. Las "gotas" o cuerpos aislados de aire polar que viajan del noreste caen en las áreas tropicales cuando chocan con el aire cálido/húmedo, mientras que las condiciones secas persisten en las áreas subtropicales y hacia el sur. Este patrón sinóptico se asemejaría a los gradientes de humedad N-S como los identificados en los datos de la cuestión paleoambiental (Messerli et al. en prensa). No obstante, poco se sabe sobre el significado estadístico y la frecuencia de tales eventos. El clima de la Fase Tauca fue posiblemente bastante más estable en una escala de 100 años con respecto a las precipitaciones (Grosjean et al. 1995), y no se sabe si los cutt-offs pudieron proveer al altiplano con precipitaciones constantes y de baja variabilidad. Sin embargo, sobreimpuestos en las precipitaciones del verano tropical pueden cumplir un papel importante en algunas áreas. Se puede especular que las grandes tormentas de baja frecuencia identificadas en la sección central de la quebrada de Puripica, con vigencia durante el Holoceno Medio, pudieron ser producidas por los eventos cutt-offs (Puna de Atacama).

Concluimos que la intensificación del monzón de verano trajo masas de vapor de agua de origen tropical/ continental hasta los $24^{\circ} \mathrm{S}$ durante el Tardío-Glacial y Holoceno Temprano. El cinturón de precipitación tropical (isoyeta $400 \mathrm{~mm}$ al año) retrocedió alrededor de los 8400 años AP de $24^{\circ} \mathrm{S}$ hasta los $16^{\circ} \mathrm{S}$, hacia el norte (lago Titicaca), produciendo un pronunciado período árido en la Puna de Atacama durante el Holoceno Medio. La transición ocurrió probablemente dentro de un corto tiempo y condujo a una caída dramática de los niveles lacustres y a un abrupto establecimiento de condiciones totalmente áridas. Este período árido fue interrumpido por grandes tormentas, pero esporádicas. El cinturón de precipitaciones monzónicas avanzó y retrocedió varios pasos de su posición actual (alrededor de los 3000 años AP). No detectamos otras evidencias tan lejanas, como algún frente occidental de precipitaciones que cumpliera un rol dominante al norte de los $27^{\circ} \mathrm{S}$ durante los últimos 10000 años. Sin embargo, debemos todavía admitir posibles 
sorpresas que están siendo documentadas con reciente información de terreno sobre esta problemática aún plenamente vigente.

\section{Algunas preguntas sin respuestas}

¿Qué factores obligaron al cinturón de precipitación monzónico avanzar y retroceder sobre la Puna de Atacama? Mecanismos externos e internos o incluso una combinación de ambos están bajo consideración, aunque la respuesta definitiva no se conoce con toda precisión.

Se cree que los parámetros Milancovitch intensificaron el monzón de verano en las áreas tropicales del hemisferio norte durante el Holoceno Temprano (Kutzbach y Street-Perrott 1985). Por el contrario, del hemisferio norte, la insolación del verano y la estacionalidad fueron mínimas en las zonas de bajas latitudes del hemisferio sur durante este período. Descubrimos a través de evidencias concretas que la Fase Tauca fue más larga en el Holoceno Temprano de lo que se había creído anteriormente y podría, por lo tanto, estar relacionada hasta cierto punto con el Holoceno Pluvial Temprano en las áreas tropicales del hemisferio norte. Podría considerarse una teleconexión a la rama nortina de la celda tropical Hadley, al menos por un período de tiempo limitado.

Un segundo tipo de fuerza externa podría identificarse a través de los patrones de circulación del océano a lo largo de la costa sudamericana. Durante parte del Holoceno Temprano el Pacífico sureste fue más cálido que hoy (Rolling et al. 1986). Precisamente, en la costa del Desierto de Atacama se identificaron siete especies de peces tropicales, en un campamento datado a los 9700 años AP (Llagostera 1979). En suma, el anticiclón del Pacífico sureste posiblemente se desestabilizó y últimamente disminuyó su poder en el bloqueo de la humedad. Al respecto, Villagrán y Varela (1990) concluyen que el aumento de la influencia del anticiclón del Pacífico sureste produjo precisamente condiciones áridas durante el Holoceno Medio en Chile Central. Tal propuesta coincide bien con la historia costera de la corriente de Humboldt, tal como se identificó en las zonas costeras peruanas (Schrades y Sakness 1991). La emergencia de aguas frías costeras fue mínima durante el comienzo del estado isotópico marino (MIS 1: Estadio marino isotópico 1), en donde la actividad de la corriente de Humboldt alcanzó el punto máximo durante la mitad del MIS 1 (Holoceno Medio).
También debemos considerar la fuerza interna del paleomonzón de la Fase Tauca debido a las variaciones de las características de la superficie y a cambios en la radiación del altiplano propiamente tal. Se espera una reducción a gran escala de los albedos de la superficie (refracción de la radiación solar), debido a las superficies más extensas de los lagos antiguos (cuenca de sal blanca/albedo = 0.7 ; lago $=0.1$ ), mientras que las superficies glaciales más extensas y capas de nieve aumentarían significativamente el albedo total de un área en particular. La diferencia neta en el albedo de la superficie entre los efectos positivos y negativos tendría que evaluarse en las áreas de estudio donde los cambios paleoambientales a través del tiempo son más conocidos.

Los argumentos fundamentales, el océano cambiante o variable, la teleconexión con el hemisferio norte y las tierras bajas continentales de Sudamérica, y los cambios internos en las características de las superficies vale la pena ser debatidos, ya que todos proporcionan un sistema adecuado para comprender la emergencia de cambios rápidos (Lorius y Oeschger 1994; Street-Perrot 1994). Sin embargo, la cubierta de nubes es uno de los elementos más variables y poderosos de la radiación, y por lo tanto del clima. Hemos destacado la alta sensibilidad de la evaporación de agua en el altiplano con respecto a la cubierta de nubes. Al respecto, Cess y colaboradores (1995) demostraron impresionantemente que los supuestos habituales de los estudios sobre la radiación de las nubes tienen que ser revisados. Agregamos: ¿Y paleonubosidad? Todo lo que sabemos ahora es que esta cuestión es muy importante, pero no podemos entenderla en términos claramente explicativos. En suma, la relación entre las poblaciones paleoindias, arcaicas y agropastoralistas tempranas y los cambios finipleistocénicos-holocénicos contrastados con este criterio geoarqueológico constituyen el curso actual de nuestras investigaciones multidisciplinarias.

Agradecimientos Este estudio fue financiado con un Grant de la Fundación Nacional de Ciencias de Suiza (NF 20-36382.92) Proyecto "Cambio climático en los Andes áridos" y del Proyecto FONDECYT 1930022. Agradecemos de modo especial a Willi Egli, Marcela Espinoza (DIFROL) y CONAF, por la ayuda logística y permisos conducentes a esta investigación y al apoyo recibido del personal técnico del IIAM, de la Universidad Católica del Norte. 


\section{REFERENCIAS CITADAS}

ARAVENA, R., H. PEÑA, A. GRILLI, O. SUZUKI y M. MORDECKAI, 1989. Evolución isotópica de las lluvias $\mathrm{y}$ origen de las masas de aire en el altiplano chileno. IAEATECDOC 502: 129-142.

CESS, R.D., M. H. ZHANG, P. MINNIS, L. CORSETTI, E. G. DUTTON, B.W. FORGAN, D. P. GARBIER, W. L. GATES, J. J. HACK, E. F. HARRISON, X. JING, J. T. KIEHL, C. N. LONG, J. MORCRETTE, G. L. POTTER, V. RAMAMATHAN, B. SUBASILAR, CH. WHITLOCK, D. F. YOUNG e Y. ZHOU, 1995. Absortion of solar radiation by clouds: Observations, versus models. Science 267: 496-503.

CLAYTON, J. D. y C. M. CLAPPERTON, Broad synchrony of a late-glacial glacier advance and the highstand of paleolake Tauca in the Bolivian altiplano. Journal of Quaternary Science. En prensa.

FUENZALIDA, H. y J. RUTLLANT, 1996. Estudio sobre el origen del vapor de agua que precipita en el invierno altiplánico. Informe final. Universidad de Chile, Santiago.

GRAF, K., 1992. Pollendiagramme aus den Anden. Eine Synthese zur Klimageschichte und Vegetation-sentwicklung seit der letzten Eiszet. Physische Geographie 34: 1-138.

GROSJEAN, M., 1994. Paleohydrology of Laguna Lejía North Chilean altiplano) and climatic implications for Late Glacial times. Paleogeography, Paleoclimatology, Palaeoecology 109: 89-100.

GROSJEAN, M. y L. NUÑEZ, 1994. Lateglacial, Early and Middle Holocene environments, human occupation and resource use in the Atacama (Northern Chile). Geoarchaeology 9: 271-286.

GROSJEAN, M., M. A. GEYH, B. MESSERLI, y U. SCHOTTERER, 1995. Late Glacial and Early Holocene lake sediments, groundwater formation and climate in the Atacama altiplano $22-24^{\circ}$ S. Journal of Paleolimnology 14: 241-252.

GROSJEAN, M., B. VALERO-GARCES, M. A. GEYH, B. MESSERLI, U. SCHOTTERER, H. SCHREIER y K. KELTS, 1997. Mid and Late Holocene limnogeology of laguna del Negro Francisco, Northern Chile, and its paleoclimatic implications. The Holocene 7 (2): 151-159.

ISACKS, B. L., 1988. Uplift of the Central Andean plateau and bending of the Bolivian orocline. Journal of Geophysical Research 93: 3211-3231.

KUTZBACH, J. E. y F. A. STREET-PERROT, 1985. Milankovitch forcing of fluctuations in the level of tropical lakes from 18 to 0 kyr BP. Nature 317: 130-134.

LEDRU, M.P., 1993. Late Quaternary environmental and climatic changes in Central Brazil. Quaternary Research 39: 90-98.

LORIUS, C. y H. OESCHGER, 1994. Paleo-perspectives: Reducing uncertainties in global change. Ambio 23/1: 30-36.

LLAGOSTERA, A., 1979. 9700 years of maritime subsistence on the Pacific and analysis by means of bioindicators in the north of Chile. American Antiquity 44: 309-324.
MARKGRAF, V., 1983. Late and postglacial vegetational and paleoclimatic changes in subantarctic temperate, and arid environments in Argentina. Palynology 7: 43-70.

MARTIN, L., M. FOURNIER, P. MOURGUIART, A. SIFEDDINE, B. TURCQ, M. K. ABSY y J. M. FLEXOR, 1993. Southern oscillation signals in south american paleoclimate data of the last 7000 years. Quaternary Research 39: 338-346.

MESSERLI, B., M. GROSJEAN, G. BON ANI, A. BÜRGI, M. A. GEYH, K. GRAF, K. RAMSEYER, H. ROMERO, U. SCHOTTERER, H. SCHREIER y M. VUILLE, 1993. Climate change and dynamics of natural resources in the Altiplano of Northern Chile during Late Glacial and Holocene times. First synthesis. Mountain Research and Development 13/20: 117-127.

MESSERLI, B., C. AMMANN, M. A. GEYH, M. GROSJEAN, B. JENNY, K. KAMMER y M. VUILLE. Forthcomingcurrent precipitation, late pleistocene snow line, lake level changes in the Atacama altiplano ( $18^{\circ} \mathrm{S}$ and $\left.28^{\circ} 30^{\prime} \mathrm{S}\right)$ : the problem of the "Andean Dry Diagonal". Bamberger Geographische Schriften.

NUÑEZ, L., 1992. Emergencia de complejidad y arquitectura jerarquizada en la Puna de Atacama: Las evidencias del sitio Tulan 54. Taller de Costa a Selva, M. E. Albeck (Ed.), pp. 85115, Universidad de Buenos Aires, San Salvador de Jujuy.

ROLLINS, H. B. III, J. B. RICHARDSON y D. H. SANDWEISS, 1986. The birth of El Niño: Geoarchaeological evidence and implications. Geoarchaeology 1: 17-28.

SCHRADER, H. y R. SORKNES, 1991. Peruvian coastal upwelling: Late Quaternary productivity changes revealed by diatoms. Marine Geology 97: 233-249.

SHUKLA, J., C. NOBRE y P. SELLERS, 1990. Amazon deforestation and climate change. Science 247: 1322-1325.

STREET-PERROTT, F. A., 1994. Paleo-perspectives: Changes in terrestrial ecosystems. Ambio 23/1: 37-43.

THOMPSON, L. G., E. P. MOSLEY-THOMPSON, J. E. BOLZANI y A. B. R. KOCI, 1985. 1500 years record of tropical precipitation recorded in ice cores from the Quelcaya ice cap, Perú. Science 225: 971-973.

VALERO-GARCES, B., M. GROSJEAN, A. SCHWALB, M. A. GEYH, B. MESSERLI y K. KELTS, 1996. Limnogeology of laguna Miscanti: Evidence for Mid to Late Holocene environmental change in the Atacama altiplano (Chile). Journal of Paleolimnology 16: 1-21.

VEIT, H., 1996. Southern weste-lies during the Holocene deduced from geomorphological and pedological studies in the Norte Chico, Northern Chile $\left(27-33^{\circ} \mathrm{S}\right)$. Palaeogeography, Palaeoclimatology, Palaeoecology 123: 107-119.

VILLAGRAN, C. y J. VARELA, 1990. Palynological evidence for increased aridity on the central chilean coast during the Holocene. Quaternary Research 34: 198-207. 